\title{
Can lycopene eliminate the harmful effects of hyperoxia in an immature brain?
}

\author{
Ahmet Ozdemir, M.D. ${ }^{a}$, Osman Bastug, M.D. ${ }^{a}$, Miss Kubra T. Cilenk ${ }^{b}$, \\ Levent Korkmaz, M.D. ${ }^{a}$, Sabriye Korkut, M.D. ${ }^{a}$, Mehmet A. Ozturk, M.D.y Prof. ${ }^{a}$ and \\ Mehmet F. Sonmez M.D., Prof.
}

\begin{abstract}
Objectives. In addition to protecting cells against free radical harm thanks toits anti-oxidant nature, lycopene strengthens the bonds among cells and improves cell metabolism. This study focuses on analyzing therapeutic effects of lycopene in hyperoxia-induced neurodegenerative disorders in newborn rats.

Methods. Term newborn rats were divided into four groups as the normoxia control group (group-1), normoxia+lycopene group (group-2), hyperoxia control group (group-3) and hyperoxia+lycopene group (group-4). Group-1 and group-2 were monitored in room air while the group- 3 and group- 4 were monitored at $>85 \% \mathrm{O}_{2}$. The group- 2 and group -4 were injected with lycopene intrapertioneally (i.p.) at $50 \mathrm{mg} /$ $\mathrm{kg}$ / day while the other groups were injected with corn oil i.p. at the same volume. The rats we sacrificed on the $11^{\text {th }}$ day following the 10-day hyperoxia. Thebrains were removed and oxidant system parameters were assessed.
\end{abstract}

Results. Injury resulting from hyperoxia was detected in the white matter, cortical regions, and thalamus of the brains. It was observed that the number of apoptotic cells increased and the number of proliferating cell nuclear antigen (PCNA) positive cells decreased in the groups-3 and 4 compared to the group- 1 . No significant improvement in the number of apoptotic cells and PCNA positive cells was observed in the groups-3 and 4, and apoptosis increased as well. Conclusion. This study found that lycopene, did not show any therapeutic effects for brain damage treatment in newborn rats. In addition, this study demonstrated that lycopene might lead to toxic effects.

Key words: free radicals, hyperoxia, brain damage, lycopene, newborn rats.

http:/ / dx.doi.org/10.5546/ aap.2019.eng.237

To cite: Ozdemir A, Bastug O, Cilenk KT, Korkmaz L, et al. Can lycopene eliminate the harmful effects of hyperoxia in an immature brain? Arch Argent Pediatr 2019;117(4):237-244.

\section{INTRODUCTION}

Funding:

None.

Conflict of interest: None.

Received: 3-29-2018 Accepted: 2-2-2019 in infants are perinatal asphyxia, unknown etiological factors lead to neurodevelopmental disorders. ${ }^{1}$ In some cases it is necessary to administer high concentrations of oxygen immediately after birth, especially in premature infants. The toxic effects of hyperoxia play role in neonatal lung disease, premature retinopathy (ROP) and the pathogenesis of abnormal neurological results in premature infants. ${ }^{2}$ Hyperoxia leads not only to neuronal and glial cell death but also to ROP and chronic pulmonary disease. In addition, in the brains of premature newborns, it contributes to the white and grey matter injury. ${ }^{3}$ Exposure to supraphysiologic oxygen concentration in neonatal period causes disorders in cerebral functions, together with neuronal system degeneration in future life. ${ }^{4}$ Experimental brain damage caused by hyperoxia was associated with oxidative stress in the brain parenchyma and reduction in neuronal density, increase of proinflammatory cytokines, proliferation of oligodendroglia and vascular cell death. ${ }^{5}$ A number of questions related to clinical data for the most suitable $\mathrm{FiO}_{2}$ in infants with excessively low birth weights and oxygen use in newborn period still remain unanswered. ${ }^{6}$ Oxidative stress triggered by hyperoxia reflected in increase in oxidized glutathione concentration and decrease in reduced glutathione as well as lipid peroxidation in immature brain. ${ }^{7}$ Accordingly, the need for more research into the supraphysiological levels of oxygen, the use of which is vital in neonatal intensive care units, remains to continue. 
Lycopene, which is naturally present in tomato and processed tomato products as well as fruitsvegetables, is a non-cyclic carotenoid which chemically contains a total of 13 double bonds (11 conjugated and 2 non-conjugated bonds). It is mostly found in low density lipoprotein and very low density lipoprotein fractions in prostate glands, testes, suprarenal glands and liver. ${ }^{8}$ Lycopene, which protects cells against free radical damage, improves cell metabolism and strengthens the bonds among cells. ${ }^{8}$

In a study on lycopene, it was found that it increased endogenous anti-oxidant activities such as glutathione peroxidase (GSPx) and superoxide dismutase (SOD), and decreased the malondialdhyde (MDA) level which is known to be related to oxidative damage and is the end product of fatty acid oxidation. ${ }^{9}$ The effect of lycopene on inflammatory indicators such as tumor necrotizing factor- $\alpha$ (TNF- $\alpha$ ), interleukin$1 \beta$ (IL-1 $\beta$ ) and interleukin-6 (IL-6) showed a downward trend, too. ${ }^{10}$
Hyperoxia causes damage to white matter associated with oxidative stress in the rat brain and this may give us an idea about the white matter damage in premature infants. ${ }^{11}$ Therefore, this study aimed to assess the neuroprotective effect of lycopene, which possesses the strongest character among anti-oxidant carotenoids, on hyperoxic brain damage in rats.

\section{MATERIALS AND METHODS}

This study was approved by Erciyes University Animal Ethics Committee. The rats were fed with pellet feed and allowed to drink water as much as they wished. The room temperature was fixed at $23 \pm 1{ }^{\circ} \mathrm{C}$ and day/night cycle of 12 hours was maintained.

Newborn rats were used to demonstrate the toxic effects of the levels of supraphysiologic oxygen on newborns. For hyperoxia-induced inflammatory damage, there exist in the literature experimental models formed in the first postnatal 14 days on condition of starting in the early days.

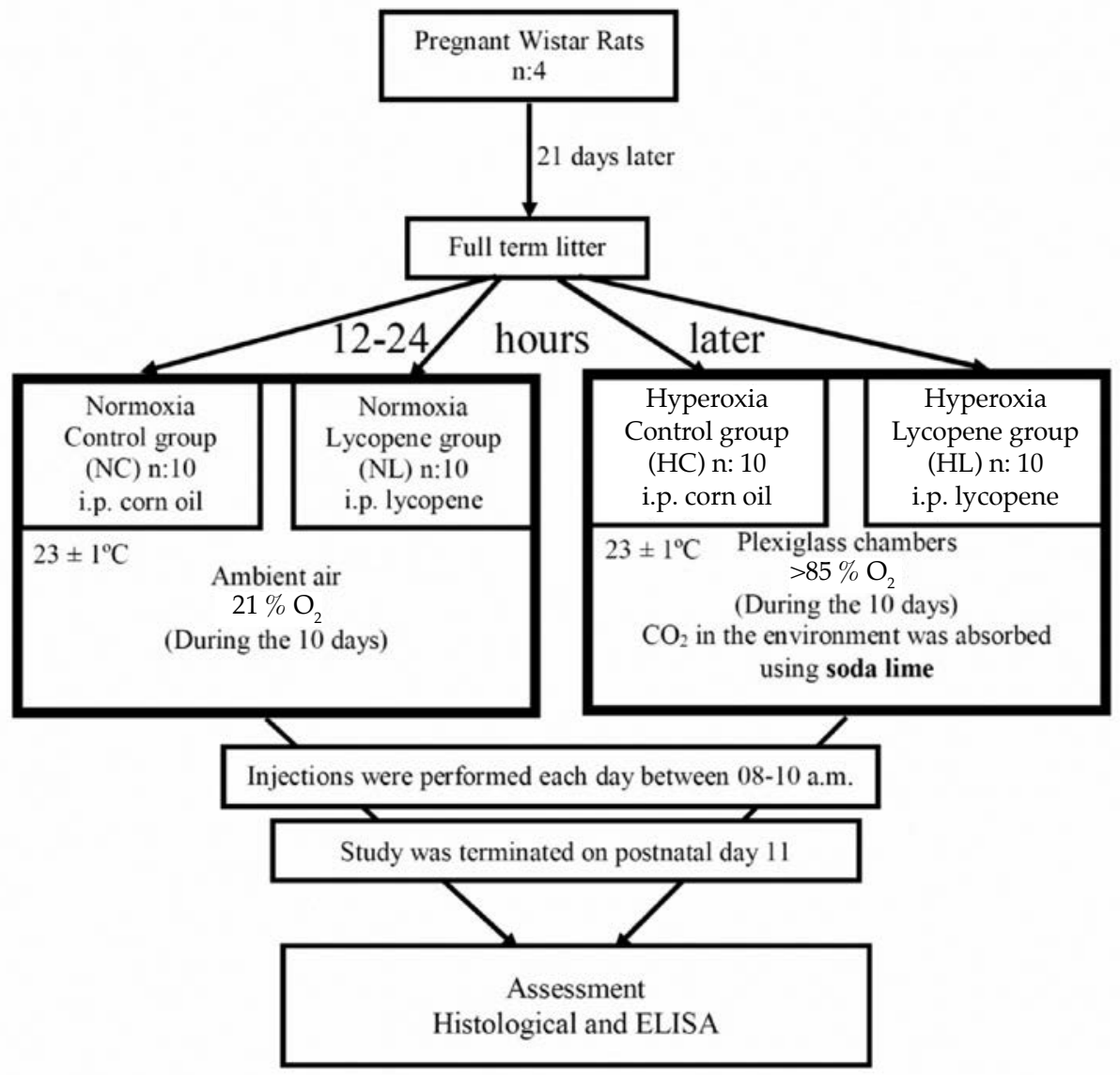


In our study, we took the experimental hyperoxia model as reference and formed the chain of inflammatory events. ${ }^{12}$ These were divided into four groups: normoxia control group (group-1), normoxia+lycopene group (group-2), hyperoxia control group (group--3) and hyperoxia+lycopene group (group-4); there were 10 newborn rats in each group.

The dosage study by Bignotto et al. ${ }^{13}$ has been taken as a basis for the anti-inflammatory effect of lycopene. All groups were injected intrapertioneally (i.p.) with $50 \mathrm{mg} / \mathrm{kg} /$ day [Lycopene, Redivivo ${ }^{\mathrm{TM}} 10 \% \mathrm{FS}, 10 \%$ in corn oil, $\geq 95.0 \%$ (sum of isomers), SIGMA, Switzerland] at the same level throughout the study. The group-1 was injected i.p. with corn oil; the group-2 was given a single dose of lycopene of $50 \mathrm{mg} / \mathrm{kg} /$ day at room temperature; the group- 3 was exposed to oxygen above $85 \%$ and injected with corn oil i.p.; and, finally, the group- 4 was given a single dose of lycopene along with oxygen above $85 \% .{ }^{12}$ The study was completed on the postnatal 11th day and the animals were sacrificed. The brain tissues were quickly removed and used for histological analysis and ELISA studies (Figure 1).

\section{Histopathological assessment}

The brain tissues were quickly removed following decapitation. Some of the tissues were fixed in neutralized formalin of $4 \%$ for 24 hours. Identified tissues were washed under tap water and dehydrated using a graded alcohol series. ${ }^{12}$ Tissues were kept in alcohol series concentrated by $50-70-80$ and $100 \%$, respectively, for one hour in each, and thus, their water content was removed to saturate the tissues with alcohol. Tissues were made transparent using xylol and embedded in paraffin. Alcohol was substituted by xylol, to allow the penetration of paraffin through the tissue. In order to assess the normal structure, sections of 5-6 $\mu \mathrm{m}$ taken from paraffin blocks were immunostained, using the TUNEL method, with hematoxylin-eosin in order to show apoptosis and with proliferating cell nuclear antigen (PCNA) in order to observe cell proliferation.

\section{TUNEL Method}

The TUNEL method was used in order to show apoptosis in the brain tissue. The "In situ Cell Death Detection Kit, Fluorescein" was used for this purpose and staining was performed according to the kit's user guide. The tissues were washed with phosphate buffer (PBS) three times for 5 minutes and were incubated with the TUNEL reaction mixture in the kit in a humid and dark setting at $37^{\circ} \mathrm{C}$ for 60 minutes. Tissues which were washed with PBS three times for 5 minutes were stained negatively with DAPI. Tissues were covered with a glycerol occlusion solution and monitored under an Olympus BX-51 fluorescent microscope at 450-500 nm wavelength. Apoptotic cells in the tissues were counted to create an apoptotic index. Images from 10 different areas were obtained from each section using $40 \mathrm{X}$ zooming for the apoptotic index. The ratio of apoptotic cells to total cell number in these areas was determined as the index.

\section{Immunohistochemistry}

The avidin-biotin-peroxidase method was used for marking in order to identify differences in PCNA expression in the brain tissue. Tissues measuring 5-6 $\mu \mathrm{m}$ were kept at $60^{\circ} \mathrm{C}$ for one night. First, the sections were dehydrated using a graded alcohol series and washed with PBS three times for 5 minutes. Afterwards, they were placed in $0.01 \mathrm{M}$ citrate buffer in a microwave oven at $600 \mathrm{~W}$ for $3 X 5$ times for antigen recovery and kept in the same buffer solution at room temperature for 20 minutes. Sections were washed with PBS again and treated with hydrogen peroxide of $3 \%$ for 5 minutes in order to prevent endogenous peroxidase activity, and an $\mathrm{ABC}$ staining system (Santa Cruz, sc-2023) staining kit was used for later stages. Sections, which were washed with PBS again, were applied with block serum at room temperature for 20 minutes in order to cover areas outside the antigenic areas. Sections were kept at $+4{ }^{\circ} \mathrm{C}$ with primary antibody for PCNA (sc-9857) for one night, and incubated at room temperature for 20 minutes on the following day. PBS was used instead of primary antibody for negative control. The sections were later treated with avidin-biotin enzyme separator for 30 minutes and washed with diaminobenzidine water in order to display immune reactivities. In the final stage, water was removed using an increasing alcohol series and the sections put in xylene were covered with entellan. Images were obtained using DP71 digital camera under Olympus BX51 light microscope. Images were obtained from five different areas in each subject. The number of PCNA positive cells was proportioned to the total cells in Image $\mathrm{J}$ and differences the between groups were displayed. 


\section{ELISA}

Homogenized brain tissues were centrifuged at $+4{ }^{\circ} \mathrm{C}$ and $12000 \mathrm{rpm}$ for 30 minutes and the remaining supernatants were aliquoted for the ELISA procedure. Later, brain tissue homogenates were analyzed using SOD (CSB-E08555r), MDA (CSB-E08558r), TNF- $\alpha$ (Invitrogen KRC3011),
IL-1 $\beta$ (Invitrogen KRC0011), IL-6 (Invitrogen KRC0061) and GSPx (Cayman-703102) ELISA kits.

\section{Statistical analysis}

All statistical analysis was performed using SPSS. Data were identified as mean \pm standard deviation. The Kruskal-Wallis test was used for

FIGURE 2. TUNEL positive cells are distinguished in brain sections. Apoptotic cell nuclei (arrows) are fluorescent green, while the other cells are fluorescent blue when stained with DAPI. (A) Normoxia control group (group 1) (B) Normoxia + lycopene group (group 2) (C) Hyperoxia control group (group 3) (D) Hyperoxia + Lycopene group (group 4).

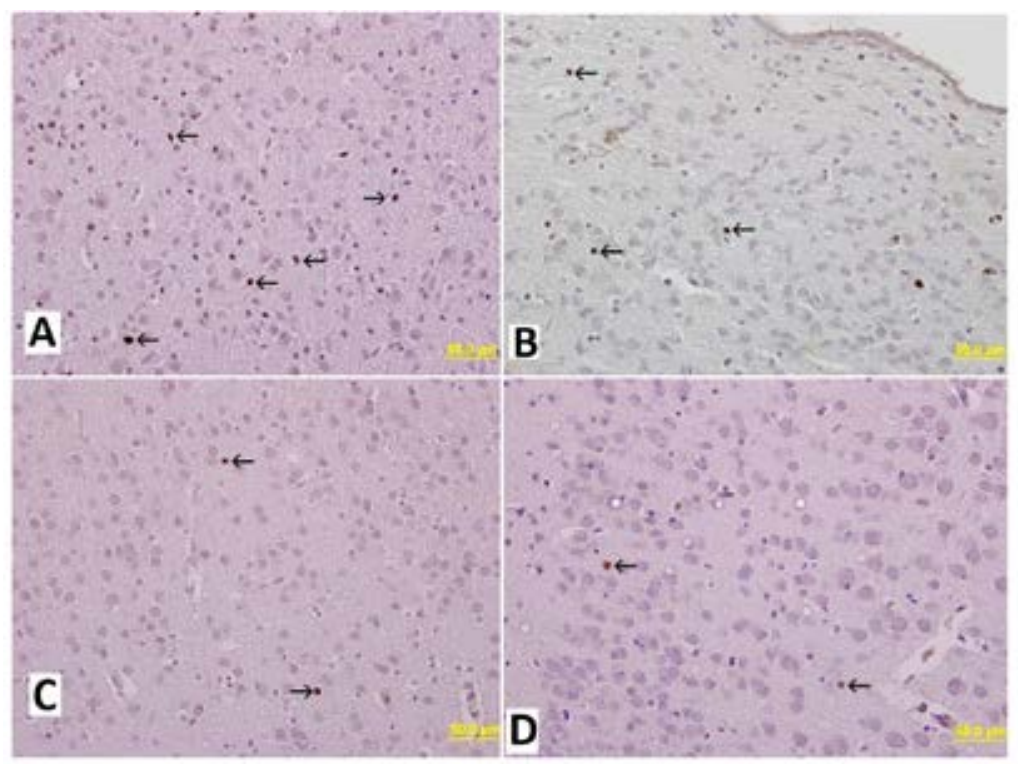

FIGURE 3. PCNA positive cells are distinguishable in brain sections (arrow). (A) Normoxia control group (group 1)(B) Normoxia + lycopene control group (group 2) (C) Hyperoxia group (group 3) (D) Hyperoxia + Lycopene group (group 4).

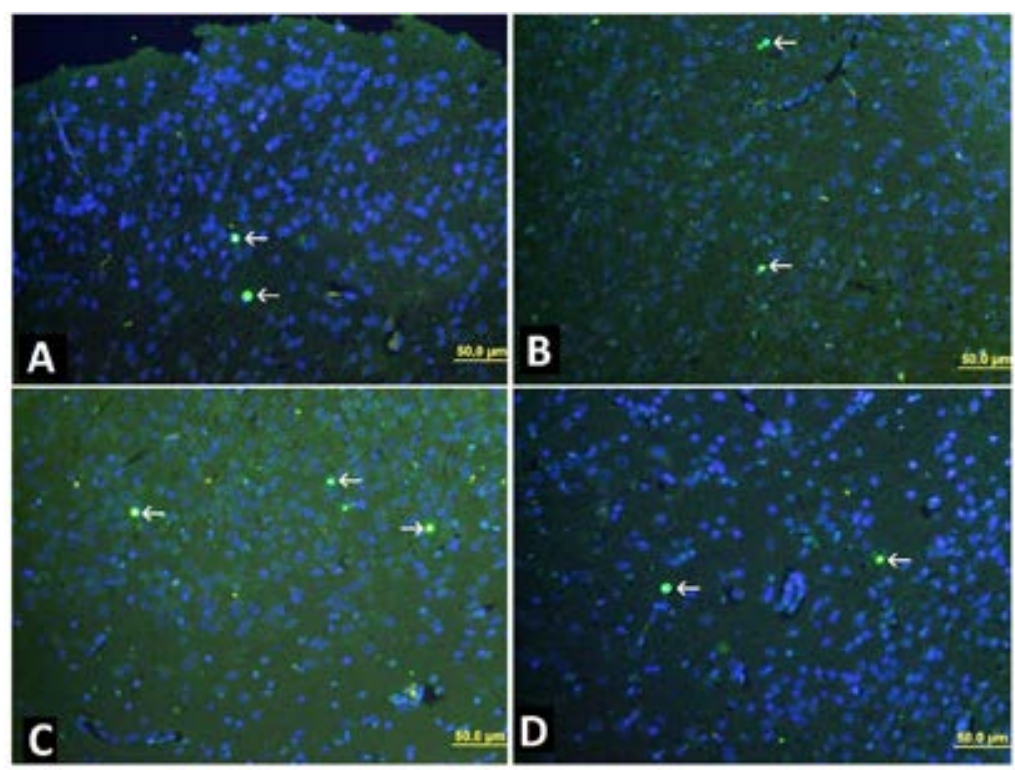


results analyzed using ELISA while the one way ANOVA method was used for apoptotic index in the brain tissue and PCNA score. Tukey test was used for post-hoc analysis. Statistical analysis was considered as $\mathrm{p}<0.05$.

\section{RESULTS}

In our study, injury resulting from hyperoxia was detected in the white matter, cortical regions, and thalamus. TUNEL method (Figure 2) was used in order to identify apoptosis in the brain sections, while PCNA (Figure 3) immunohistochemistry was used in order to display cell proliferation. While the apoptotic index increased in groups-3 and 4 compared to the group-1, the number of PCNA positive cells showed a statistically significant decrease compared to the group-1. Nevertheless, an unexpected statistically significant increase was observed in the number of apoptotic cells in groups-3 and 4 compared to the group- 1 and there was a decrease in the number of PCNA positive cells (Table 1).
In order to measure the hyperoxic damage in the brain tissues, ELISA kits were used to determine MDA, SOD and GSPx, which are among oxidant system parameters, and IL- $1 \beta$, IL- 6 and TNF- $\alpha$, which are among inflammatory system indicators (Table 2). It was found that MDA, SOD and IL- $1 \beta$ increased and GSPx and TNF- $\alpha$ decreased compared to the group-1.

\section{DISCUSSION}

Hyperoxic brain damage caused by oxygen use in newborn intensive care units has recently attracted attention. Premature infants are more vulnerable to brain damage because their antioxidant defense mechanisms are not mature enough. Various studies demonstrated that hyperoxia caused oxidative and nitrative stress, directly influenced the gray and white matter areas in the brain, high oxygen concentration had harmful effects in the immature brain. ${ }^{11,14}$ Free oxygen radicals and caspase activation caused by hyperoxia trigger apoptosis in the brain during its

TABLE 1. Proliferating cell nuclear antigen and apoptotic indices in brain tissue

\begin{tabular}{lcccc}
\hline & Group 1 & Group 2 & Group 3 & Group 4 \\
\hline Apoptotic index & $0.0026 \pm \pm 0.0007$ & $0.0071 \pm 0.0023^{*}$ & $0.0045 \pm 0.0008$ & $0.0047 \pm 0.0006$ \\
PCNA index & $9.18 \pm 0.68$ & $2.11 \pm 0.31^{*}$ & $1.88 \pm 0.21^{*}$ & $1.73 \pm 0.28^{*}$ \\
\hline
\end{tabular}

Values are expressed as mean \pm standard deviation.

${ }^{*}$ Compared with the control $\mathrm{p}<0.05$.

Group 1: Normoxia control group, Group 2: Normoxia lycopene group.

Group 3: Hyperoxia control group, Group 4: Hyperoxia+lycopene group.

PCNA: Proliferating cell nuclear antigen.

TABLE 2. SOD, MDA, GSPX, TNF- $\alpha, I L-1 \beta$ and IL-6 levels in brain tissue

\begin{tabular}{lcccc}
\hline & Group 1 & Group 2 & Group 3 & Group 4 \\
\hline SOD (mlU/ml) & $5.80 \pm 2.56$ & $4.71 \pm 1.55$ & $8.56 \pm 3.00$ & \\
& $1.46(1.00-27.77)$ & $3.80(1.38-19.48)$ & $4.23(1.16-30.20)$ & $1.84(1.21-31.38)$ \\
MDA (pmol/ml) & $237.53 \pm 16.84$ & $256.76 \pm 18.08$ & $251.27 \pm 20.44$ & $211.61 \pm 15.21$ \\
& $215.82(146.14-311.95)$ & $282.66(143.11-335.76)$ & $235.15(158.97-362.59)$ & $207.85(135.72-302.28)$ \\
GSPx (nmol/min/ml) & $13.54 \pm 1.94$ & $12.34 \pm 2.11$ & $10.90 \pm 2.39$ & $14.42 \pm 1.31$ \\
& $15.79(2.04-20.63)$ & $11.20(0.76-22.92)$ & $10.18(3.82-21.14)$ & $13.75(9.93-22.41)$ \\
IL-1 $\beta$ (pg/ml) & $37.87 \pm 3.92$ & $31.35 \pm 2.38$ & $41.39 \pm 5.14$ & $35.99 \pm 3.01$ \\
& $34.37(20.48-65.69)$ & $31.37(17.94-42.85)$ & $34.30(22.72-76.91)$ & $34.63(25.30-56.31)$ \\
IL-6 $(\mathbf{p g} / \mathbf{m l})$ & $132.59 \pm 17.97$ & $109.70 \pm 18.90$ & $132.71 \pm 26.01$ & $96.27 \pm 15.67$ \\
& $139.08(31.37-236.49)$ & $103.98(35.35-207.35)$ & $111.98(45.97-267.05)$ & $101.44(10.07-191.08)$ \\
TNF- $\alpha(\mathbf{p g} / \mathbf{m l})$ & $97.03 \pm 10.59$ & $89.39 \pm 9.73$ & $83.16 \pm 8.02$ & $78.89 \pm 8.23$ \\
& $102.15(31.96-144.23)$ & $76.15(47.13-164.05)$ & $82.27(44.25-121.66)$ & $67.58(46.14-127.98)$ \\
\hline
\end{tabular}

Values are expressed as mean \pm standard deviation, median (minumum and maximum).

SOD: Superoxide dismutase, MDA: Malondialdehyde, TNF- $\alpha$ : Tumor necrosis factor- $\alpha$,

IL-1 $\beta$ : Interleukin-1 $\beta$, IL-6: Interleukin-6, GSPx: Glutathione peroxidase

Group 1: Normoxia control group, Group 2: Normoxia lycopene group.

Group 3: Hyperoxia control group, Group 4: Hyperoxia+lycopene group. 
maturation and cause white matter damage. ${ }^{11}$ In addition, hyperoxia-induced cell death influences the cortex, basal ganglia, hypothalamus and hippocampus. ${ }^{14}$ In our study also, hyperoxic injury was detected in the white matter of the brain as demonstrated by Gestner et al. ${ }^{11}$

Neuronal density is reduced and apoptosis increases in various areas of the brain. ${ }^{5,14}$ One of the mechanisms that contributes to hyperoxiainduced apoptosis in developing brain is oxidative stress. On the other hand, neural survival during hyperoxia exposure decreases control gene expression and protein phosphorylation. ${ }^{14}$ PCNA, which is defined as antigen expressed by the nucleus in the DNA synthesis, is important for DNA synthesis and repair and was used as an indicator of cell proliferation in our study. ${ }^{15}$ In this study, too, hyperoxic brain damage was created as shown in the method section. An increase in apoptosis and a decrease in the PCNA index were observed in the groups- 2 and $3 \mathrm{~A}$; a decrease in the GSPx, an endogenous anti-oxidant, and an increase in the MDA level, the end-product of fatty acid oxidation, were observed. In addition, TNF- $\alpha$, IL-1 $\beta$ and IL-6, which are inflammatory indicators, also were increased.

Newborn rats, which were subject to hyperoxic brain damage using existing findings, received lycopene treatment. Lycopene is an important carotenoid and possesses free radical scavenger capacity, which enables anti-oxidant defense mechanisms to protect the cell against DNA damage.$^{16}$ There are studies in the literature which evaluate the anti-oxidant feature of lycopene. ${ }^{9,17}$ However, this is the first study to have analyzed the anti-oxidant feature of lycopene in terms of hyperoxic brain damage in newborn rats.

It was identified in this study that the apoptotic index increased in the group- 4 compared to the group 1 ( $\mathrm{p}<0.05)$. In addition, it was observed that lycopene decreased the PCNA index in the brains of newborn rats exposed to hyperoxia compared to the group $1(\mathrm{p}<0.05)$. Thus, lycopene influenced cell proliferation negatively both in the groups- 2 and 4 . Although it was observed in this study that GSPx levels decreased in the group 3 and increased in the group-4 to the same level as the group-1, this wasn't statistically significant increase ( $p>0.05)$. In their experimental study analyzing the reactions of brain cells against hyperoxic conditions, Lushchak et al., ${ }^{18}$ demonstrated that the levels ofanti-oxidant enzymes SOD, catalase and GSPx, as in our study, didn't increase. In a study by Aspberg et al., ${ }^{19}$ SOD activity and mRNA levels did not change while GSPx significantly decreased in the 12th hour of hyperoxia and decrease in glutathione-reductase and glucose6-phosphate-dehydrogenase activities was observed. Furthermore, similar to various studies in the literature, inflammatory indicators such as TNF- $\alpha$, IL- $1 \beta$ and IL- 6 decreased although it wasn't a statistically significant decrease. ${ }^{9,17}$ Contrary to the data in the literature, MDA, the end-product of fatty acid oxidation, was increased in this study. ${ }^{8}$ Previous studies of $50 \mathrm{mg} / \mathrm{kg}$ of lycopene has been shown to be beneficial in gastro-esophageal reflux, acute lung injury, acute pancreatitis and hepatic ischemic reperfusion injury. ${ }^{20,21,13}$ However none of these studies was performed in newborn rats. In the studies focusing on lycopene toxicity, rats received lycopene $2 \mathrm{~g} / \mathrm{kg} /$ day for 28 days and patients received $200 \mathrm{mg} / \mathrm{kg} /$ day, which created no toxic effects. ${ }^{22}$ Similarly, a different study which lasted thirteen weeks yielded similar results. ${ }^{23}$ Although many studies regarding the benefits of lycopene are available in the literature, its harmful effects have not as yet been fully studied. In an experimental study focusing on toxicity, it was demonstrated that lycopene might function as pro-oxidant based on its dose and that lycopene protected against cell damage at physiological level (1-3 microM) while it might lead to cell loss when given at high doses $(4-10$ microM $) .{ }^{24} \mathrm{No}$ study reported the toxic effect of lycopene on the human body. ${ }^{25,26}$ The apparent brain damage in the group-4 in this study, as shown by the results of this study, may be attributed to the harmful effect caused by the high level of lycopene in the cell.

In their experimental study which focused on the toxic effects of vitamin A, de Oliveira et al. ${ }^{27}$ gave rats retinyl palmitate (vitamin A) $500 \mathrm{IU} /$ $\mathrm{kg}(\mathrm{n}=10), 1000 \mathrm{IU} / \mathrm{kg}(\mathrm{n}=10)$ and $2500 \mathrm{IU} / \mathrm{kg}$ $(n=10)$ for 3 months and analyzed mitochondrial and total 3-nitrotyrosine, monoamine oxidase and glutathione-S-transferase activities. As a result, they observed oxidative and nitrosative stress in the mitochondrial areas of the brain and reported that the group that received $500 \mathrm{IU} /$ $\mathrm{kg}$ might be reliable and that other doses might display toxic effects. Previous studies also yielded similar results. ${ }^{28,29}$ There are also studies which focused on the toxicity of vitamin A and these yielded results showing fontanel tension at higher doses. ${ }^{30,31}$ Given that lycopene belongs to the carotenoid group, which is an important 
vitamin A source, it can be argued that higher doses of lycopene are likely to be toxic..$^{32}$ This study already demonstrated that higher doses of lycopene increased apoptosis and it didn't sufficiently improve oxidant system parameters. Therefore, it is very important to determine the optimum lycopene dose. However, we consider that the lycopene dose of $50 \mathrm{mg} / \mathrm{kg} /$ day used in this study is high. It can have harmful effects rather than eliminate the effect of hyperoxia. Therefore, it should be used with caution.

\section{CONCLUSION}

This study demonstrated that lycopene didn't improve the harmful effects caused by hyperoxia and that it also caused similar cytologic and biochemical changes.

\section{Acknowledgements}

This study was supported by Erciyes University Faculty of Medicine Committee of Scientific Research Projects (Project code: TTU2013-4617).

\section{REFERENCES}

1. HintzSR, KendrickDE, VohrBR, PooleWK, et al. Changes in neurodevelopmental outcomes at 18 to 22 months' corrected age among infants of less than 25 weeks' gestational age born in 1993-1999. Pediatrics. 2005; 115 (6):1645-51.

2. Deulofeut R, Critz A, Adams-Chapman I, Sola A. Avoiding hyperoxia in infants $<$ or $=1250 \mathrm{~g}$ is associated with improved short- and long-term outcomes. J Perinatol. 2006; 26 (11):700-5.

3. Reich B, HoeberD, BendixI, Felderhoff-MueserU.Hyperoxia and the Immature Brain. Dev Neurosci. 2016; 38 (5):311-30.

4. Deuber C, Terhaar M. Hyperoxia in very preterm infants: a systematic review of the literature. J Perinat Neonatal Nurs. 2011; 25 (3):268-74.

5. Yis U, Kurul SH, Kumral A, Cilaker S, et al. Hyperoxic exposure leads to cell death in the developing brain. Brain Dev. 2008; 30 (9):556-62.

6. SaugstadOD.Hyperoxiain the term newborn:more evidence is still needed for optimal oxygen therapy. Acta Paediatr. 2012; 101 (464):34-8.

7. Sifringer M, Brait D, Weichelt U, Zimmerman G, et al. Erythropoietin attenuates hyperoxia-induced oxidative stress in the developing rat brain. Brain Behav Immun. 2010; 24 (5):792-9.

8. AgarwalS, Rao AV. Tomato lycopene and its role in human health and chronic diseases. CMAJ. 2000; 163 (6):739-44.

9. HeQ,Zhou W, Xiong C, Tan G, Chen M. Lycopeneattenuates inflammation and apoptosis in post-myocardial infarction remodeling by inhibiting the nuclear factor- $\kappa \mathrm{B}$ signaling pathway. Mol Med Rep. 2015; 11 (3):374-8.

10. Keller M, Felderhoff-Mueser U, Lagercrantz H, Dammann $\mathrm{O}$, et al. Policy benchmarking report on neonatal health and social policies in 13 European countries. Acta Paediatr. 2010; 99 (11):1624-9.

11. Gerstner B, DeSilva TM, Genz K, Armstrong A, et al. Hyperoxia causes maturation-dependent cell death in the developing white matter. J Neurosci. 2008; 28 (5):1236-45.

12. Kose M, Bastug O, Sonmez MF, Per S, et al. Protective effect of vitamin D against hyperoxia-induced lung injury in newborn rats. Pediatr Pulmonol. 2017; 52 (1):69-76.

13. Bignotto L, Rocha J, Sepodes B, Eduardo-Figueira M, et al. Anti-inflammatory effect of lycopene on carrageenaninduced paw oedema and hepatic ischaemia-reperfusion in the rat. Br J Nutr. 2009; 102 (1):126-33.

14. Felderhoff-Mueser U, Bittigau P, Sifringer M, Jarosz B, et al. Oxygen causes cell death in the developing brain. Neurobiol Dis. 2004; 17 (2):273-82.

15. Essers J, Theil AF, Baldeyron C, van Cappellen WA, et al. Nuclear dynamics of PCNA in DNA replication and repair. Mol Cell Biol. 2005; 25 (21):9350-9.

16. Aydin S, Palabiyik SS, Erkekoglu P, Sahin G, et al. The carotenoid lycopene protects rats against DNA damage induced by ochratoxin A. Toxicon. 2013; 73:96-103.

17. Stahl W, Sies H. Lycopene: a biologically important carotenoid for humans? Arch Biochem Biophys. 1996; 336 (1):1-9.

18. Lushchak VI, Bagnyukova TV, Husak VV, Luzhna LI, et al. Hyperoxia results in transient oxidative stress and an adaptive response by antioxidant enzymes in goldfish tissues. Int J Biochem Cell Biol. 2005; 37 (8):1670-80.

19. Aspberg A, TottmarO.Oxidativestress decreases antioxidant enzyme activities in reaggregation cultures of rat brain cells. Free Radic Biol Med. 1994; 17 (6):511-6.

20. Giri AK, Rawat JK, Singh M, Gautam S, Kaithwas G. Effect of lycopene against gastroesophageal reflux disease in experimental animals. BMC Complement Altern Med. 2015; 15:110.

21. OzkanE,AkyüzC, DulunduE, Topaloglu U, etal. Protective effects of lycopene on cerulein-induced experimental acute pancreatitis in rats. J Surg Res. 2012; 176 (1):232-8.

22. Jian WC, Chiou MH, Chen YT, Lin CN, et al. Twentyeight-day oral toxicity study of lycopene from recombinant Escherichia coli in rats. Regul Toxicol Pharmacol. 2008; 52 (2):163-8.

23. MellertW, DeckardtK, GembardtC,SchulteS, et al. Thirteenweek oral toxicity study of synthetic lycopene products in rats. Food Chem Toxicol. 2002; 40 (11):1581-8.

24. Lowe GM, Booth LA, Young AJ, Bilton RF. Lycopene and beta-carotene protect against oxidative damage in HT29 cells at low concentrations but rapidly löse this capacity at higher doses. Free Radic Res. 1999; 30 (2):141-51.

25. Karppi J, Laukkanen JA, Sivenius J, Ronkainen K, Kurl S. Serum lycopene decreases the risk of stroke in men: a population-based follow-up study. Neurology. 2012; 79 (15):1540-7.

26. Renju GL, Muraleedhara Kurup G, Bandugula VR. Effect of lycopene isolated from Chlorella marina on proliferation and apoptosis in human prostate cancer cell line PC-3. Tumour Biol. 2014; 35 (11):10747-58.

27. de Oliveira MR, da Rocha RF, Pasquali MA, Moreira JC. The effects of vitamin A supplementation for 3 months on adult rat nigrostriatal axis: increased monoamine oxidase enzyme activity, mitochondrial redox dysfunction, increased $\beta$-amyloid (1-40) peptide and TNF- $\alpha$ contents, and susceptibility of mitochondria to an in vitro $\mathrm{H} 2 \mathrm{O} 2$ challenge. Brain Res Bull. 2012; 87 (4-5):432-44.

28. Calabrese V,Mancuso C, Calvani M, Rizzarelli E, et al. Nitric oxide in the central nervous system: neuroprotection versus neurotoxicity. Nat Rev Neurosci. 2007; 8 (10):766-75.

29. de Oliveira MR, Moreira JCF. Acute and chronic vitamin A supplementation at therapeutic doses induces oxidative stress in submitochondrial particles isolated from cerebral cortex and cerebellum of adult rats. Toxicol Lett. 2007; 173 (3):145-50.

30. West KP Jr, Khatry SK, LeClerq SC, Adhikari R, et al. Tolerance of young infants to a single, large dose of vitamin A: a randomized community trial in Nepal. Bull World Health Organ. 1992; 70 (6):733-9.

31. oral dose of vitamin A administered to neonates. Bull World Health Organ. 1994; 72 (6):859-68.

32. Zhu YH, Jiang JG, Chen Q. Characterization of cDNA of lycopene beta-cyclase responsible for a high level of betacarotene accumulation in Dunaliella salina. Biochem Cell Biol. 2008; 86 (3):285-92. 